\title{
Numerical computation of a confined sediment-water mixture in uniform flow
}

\author{
L. Sarno ${ }^{1}$, R. Martino ${ }^{2} \&$ M. N. Papa ${ }^{3}$ \\ ${ }^{1}$ Private Consultant, Naples, Italy \\ 2 Department of Hydraulic and Environmental Engineering 'G.Ippolito', \\ Naples University, Italy \\ ${ }^{3}$ Department of Civil Engineering, Salerno University, Italy
}

\begin{abstract}
The purpose of this paper is to simulate a laminar mud flow confined in a narrow rectangular open channel. The flow bed is an erodible layer made up of the same material involved in the flow; the equilibrium condition between the moving and non-moving layer is assumed. The mud mixture under study is ruled by the Herschel-Bulkley's (H-B) shear thickening rheological law. It is supposed that the local volumetric concentration is linearly increasing with the depth and it is constantly equal to its maximum value where the local velocity is smaller than a threshold value. Relations among rheological parameters and concentration have been obtained through laboratory rheometric tests. Turbulence effects and Coulombian stresses have been ignored. The momentum equation has to be integrated along the flow cross section for the flow velocity to be obtained. Unfortunately, it is very difficult to integrate this equation using $\mathrm{H}-\mathrm{B}$ rheological law, since there are different stress functions and it is not possible to know a priori the sub-domains of them (plug, non-plug and bed regions). In the present work a modified rheological law, continuous over the whole domain of integration is employed and the momentum equation is numerically integrated. This modified law has been obtained by adding a constant correcting the denominator in the $\mathrm{H}-\mathrm{B}$ stress functions. Therefore, there are no longer any dead zones or plug regions. However it is noteworthy that, using a small constant, the model produces a good simulation of plug and dead zones: i.e. the velocity gradient is very small there. The mathematical model has two parameters: maximum concentration and threshold velocity. These parameters have been adjusted by back-analysis with measurements from laboratory flume experiments in uniform flow conditions. Keywords: mud flow, Herschel-Bulkley rheological law, equilibrium, plug.
\end{abstract}




\section{Introduction}

Mud flows are very dangerous for anthropic settlements and so, during the last years, they have been widely studied in environmental engineering. Mud flow is characterized by motion of a two-phase mixture, consisting of water and highconcentrated fine-granulometry solid matter; therefore, its mechanical behavior is solid-like while the acting shear stresses are smaller than a fixed yield stress, and it is similar to a non-Newtonian fluid when the acting stresses are bigger. Researcher aim at obtaining a resistance law correlating the flow rate and the flow depth, taking into account both the natural mixtures and irregular-shape flow cross sections.

The present work presents a computational model to study the mud flow under some simplifying hypotheses. The numerical computation was performed in MATlab environment, by implementing a finite-difference method.

\section{Constitutive law and definitions}

There are two ways to approach the mechanical problem;

1. considering independently solid and liquid phases

2. using an equivalent fluid model with a rheological law, which, taking into account all the modes of resistance inside the mixture, relates the shear stress $\tau$ with the shear rate $\dot{\gamma}$.

In this work the problem is tackled according to the second approach, i.e. a rheological law, where parameters depend on the local volumetric concentration of solid matter, has been used. The mixture ability to support shear stress depends on the relative distance existing between solid particles and, consequently, on the solid concentration.

The motion, under study, takes place in a rectangular-shaped open channel. Uniform and laminar conditions have been assumed: that means the velocity is a scalar function defined over the flow cross section. The motion develops on erodible layer, consisting of the same solid matter which is in the mixture, and in equilibrium condition: there is a dynamic equilibrium between solid deposit and particles at motion inside the flow. Therefore, at equilibrium there is a zone, called dead zone, where solid matter is at rest. To tell the cross section sub-domain, where velocity is non-null, from the whole cross section, the first one will be called "active cross section" and the other one simply "cross section". The velocity function, in equilibrium condition, is marked out by null gradient at the boundary, between active flow cross section and dead zone.

The following anticlockwise system of axes has been assumed as reference frame: the $x$ axis is parallel to the motion direction and so it is perpendicular to the cross section, $y$ is perpendicular to the erodible bed and it lies on the cross section, $z$ is parallel to the absolute bed plane and it lies on the cross section.

A scheme of the channel and the reference frame is reported in fig. 1. 


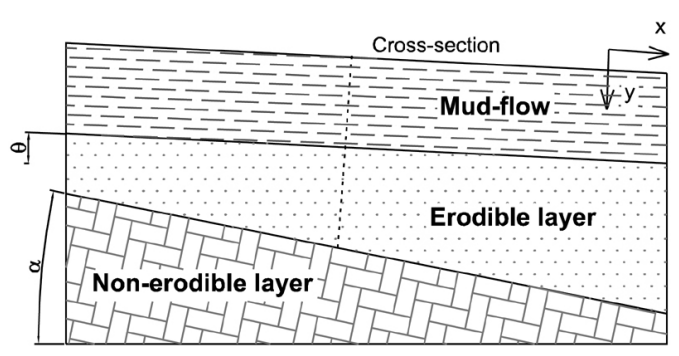

Figure 1: View of $x y$ plane, $z$-axis is perpendicular to the sheet. It is shown that usually erodible layer slope is different from flume bed slope. The dashed line represents the cross section.

The mixture considered is ruled by Herschel-Bulkley's law, which presents the following one-dimensional form:

$$
\begin{array}{ll}
\tau=\tau_{B}+\mu\left(\frac{d u}{d n}\right)^{\eta} & \text { if } \tau>\tau_{B} \\
\frac{d u}{d n}=0 & \text { if } \tau \leq \tau_{B}
\end{array}
$$

where $u$ is the velocity, $\tau$ the shear stress, $\tau_{B}$ the yield stress, $\mu$ the apparent viscosity. The second form is due to the fact that, when acting stress is smaller than yield stress, the behavior of mixture is solid-like.

In steady conditions, everywhere the acting shear stress is equal to the resistant one.

The general expression of H-B's law [1], valid for three-dimensional problems, is the following one:

$$
\begin{array}{ll}
\mathbf{T}-p \mathbf{I}=\frac{\tau_{B} \mathbf{D}}{\sqrt{-D_{I I}}}+\frac{2^{\eta} \mu \mathbf{D}}{\sqrt{-D_{I I}^{1-\eta}}} & \text { if } \tau>\tau_{B} \\
\mathbf{D}=0 & \text { if } \tau \leq \tau_{B}
\end{array}
$$

where $\mathbf{T}$ is the stress tensor, $\mathbf{I}$ the unit tensor, $\mathbf{D}$ the strain rate tensor, $D_{I I}$ the second invariant of the secular equation associated with tensor $\mathbf{D}$.

There are two different rheological forms: the second one postulating that, where acting stress is smaller than yield stress $\tau_{B}$, there is no strain, i.e. the whole shear stress is supported by the solid matter. The presence of a plug region, where the velocity vector is constant, set on the top of the flow, close to the free surface, is a direct consequence of the above.

Fig. 2, reproduced from [2], shows an example of a typical velocity distribution, where dead and plug zones can be seen. 


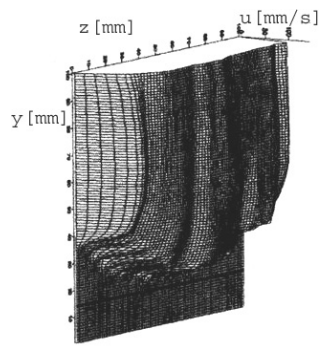

Figure 2: Velocity distribution, observed in laboratory flume experiments: dead and plug zones are evident. [2].

Rheological parameters $\tau_{B}$ and $\mu$ strongly depend on the local concentration, but $\eta$ is only dependent on the chemical-physical nature of the solid suspension. For solid matter used in laboratory flume experiments, coming from the area of Sarno (Italy), the following fitting forms have been obtained, through rheometric tests:

$$
\tau_{B}=0.0589 \cdot e^{12.071 \cdot c}, \mu=0.0020 \cdot e^{9.382 \cdot c}, \eta=1.722
$$

where $c$ is the local volumetric concentration. This dependence is exponential, therefore a good estimation of $c$ is essential.

In this paper three kinds of concentration have been used: volumetric local concentration $c$, mean concentration $c_{m}$ over the cross section and flow concentration $c_{t}$. They are defined in the following expressions:

$$
c(\mathbf{x}):=\lim _{V \rightarrow 0} \frac{V_{s}}{V_{s}+V_{w}}, c_{m}:=\frac{\int_{\Omega} c(\mathbf{x}) d A}{\Omega}, c_{t}:=\frac{\int_{\Omega} c(\mathbf{x}) \mathbf{u}(\mathbf{x}) \cdot \hat{\mathbf{n}} d A}{\int_{\Omega} \mathbf{u}(\mathbf{x}) \cdot \hat{\mathbf{n}} d A}
$$

where $V_{s}$ the volume taken up by the solid suspension, $V_{w}$ the volume of water. $\Omega$ is the cross section domain, $\mathbf{u}(\mathbf{x})$ the velocity at $\mathbf{x}$ point, $\hat{\mathbf{n}}$ the unit vector normal to the cross section.

The local concentration is defined over the whole section, whereas the mean concentration and the flow concentration are features of the entire motion. The flow concentration means also the ratio between the solid flow rate and the total one.

Projecting on $x$-axis eqn (3), the following expressions have been obtained:

$$
\tau_{y x}=\frac{\tau_{B}+\mu\left[\left(\frac{\partial u}{\partial y}\right)^{2}+\left(\frac{\partial u}{\partial z}\right)^{2}\right]^{\frac{\eta}{2}}}{\sqrt{\left[\left(\frac{\partial u}{\partial y}\right)^{2}+\left(\frac{\partial u}{\partial z}\right)^{2}\right]}} \frac{\partial u}{\partial y}
$$




$$
\tau_{z x}=\frac{\tau_{B}+\mu\left[\left(\frac{\partial u}{\partial y}\right)^{2}+\left(\frac{\partial u}{\partial z}\right)^{2}\right]^{\frac{\eta}{2}}}{\sqrt{\left[\left(\frac{\partial u}{\partial y}\right)^{2}+\left(\frac{\partial u}{\partial z}\right)^{2}\right]}} \frac{\partial u}{\partial z},
$$

where $\tau_{z x}$ is the $x$-axis component of the shear stress vector, acting on the surface with normal $z$, and similarly $\tau_{y x}$ is the $x$-axis component of the one, acting on the surface with normal $y$.

\section{Differential problem}

Momentum equation, valid everywhere over the cross section domain, can be written as:

$$
\rho(\mathbf{g}-\dot{\mathbf{u}})=\nabla \cdot \mathbf{T}
$$

where $\dot{\mathbf{u}}$ is the Lagrangian acceleration and $\mathbf{g}$ the gravity constant.

The $x$-axis component of eqn (9) can be written as follows:

$$
\rho g \sin \theta+\frac{\partial \tau_{z x}}{\partial z}+\frac{\partial \tau_{y x}}{\partial y}=0,
$$

where $\rho$ is the mean density $\left(\rho=c \rho_{\text {solid }}+(1-c) \rho_{\text {water }}\right)$ and $\theta$ is the flow slope, usually different from that of non-erodible layer, which lies below.

If velocity boundary conditions and local concentration distribution were known, since functions $\tau_{z x}$ and $\tau_{y x}$ depend on $u$ because of eqns (7) and (8), it would be possible to solve numerically the differential problem, associated to eqn (10), for the only function $u(y, z)$.

The domain of integration was a reference cross section, arbitrarily chosen in the whole flume. It is assumed that solution does not vary with total flow depth, if Coulombian stresses can be ignored.

\subsection{Corrective term $\epsilon^{2}$}

The main difficulty in integrating eqn (10) derives from its being a free-boundary problem: the size of plug sub-domain and the velocity value in the plug cannot be a priori fixed. Besides, eqn (10) is not defined in the plug. To overcome the problem, the functions (7) and (8) were replaced by the following ones, which are defined and continuous over the whole cross section:

$$
\tau_{y x}=\frac{\tau_{B}+\mu\left[\left(\frac{\partial u}{\partial y}\right)^{2}+\left(\frac{\partial u}{\partial z}\right)^{2}\right]^{\frac{\eta}{2}}}{\sqrt{\left[\epsilon^{2}+\left(\frac{\partial u}{\partial y}\right)^{2}+\left(\frac{\partial u}{\partial z}\right)^{2}\right]}} \frac{\partial u}{\partial y}
$$




$$
\tau_{z x}=\frac{\tau_{B}+\mu\left[\left(\frac{\partial u}{\partial y}\right)^{2}+\left(\frac{\partial u}{\partial z}\right)^{2}\right]^{\frac{\eta}{2}}}{\sqrt{\left[\epsilon^{2}+\left(\frac{\partial u}{\partial y}\right)^{2}+\left(\frac{\partial u}{\partial z}\right)^{2}\right]}} \frac{\partial u}{\partial z} .
$$

The critical effect, due to the $\epsilon^{2}$, is the absence of yield stress, therefore there are not dead or plug zones any more [3]. The constant $\epsilon^{2}$ should be as small as possible, for a Bingham fluid in [4] a value smaller than $10^{-16}$ is recommended. Obviously, the smaller is $\epsilon^{2}$, the closer come the expressions (11) and (12) to the original H-B's law and the more the $u$ solution will have a zone, where the velocity is almost null with its gradient, and a zone with almost constant velocity. In this paper it has been used an $\epsilon^{2}=10^{-3}$.

\subsection{Concentration distribution}

Unfortunately, it is not yet possible to obtain a reliable estimation of local concentration $c$ through experimental measures, therefore hypotheses about it should be formulated. Undoubtedly, $c$ is increasing with the depth and there are some experimental results that confirm a nearly linear trend of $c$ at solid boundary [5]. A good estimation of $c$ is essential, since rheological parameters depend on it.

In this work following hypotheses have been assumed:

- concentration linearly increasing with the depth: $c=c_{0}+k y$;

- existence of a maximum packing value of concentration $c_{\max }$, independent of parameters of motion which vary from case to case (e.g. slope, $c_{m}$ );

- existence of a threshold value of velocity $u_{t h r}$, under which concentration is equal to maximum packing value.

Values between $[0.66-0.69]$ for $c_{\max }$ and between $\left[10^{-4}-10^{-3} \mathrm{~m} / \mathrm{s}\right]$ for $u_{t h r}$ were tried.

\subsection{Boundary conditions}

The following boundary conditions have been assumed:

- no-slip condition, that is null velocity everywhere at solid boundaries of channel;

- null shear stress at free surface.

The model could be tested also in slip condition at side solid border, which seems to be more realistic, but it is very difficult to obtain a reliable experimental estimation of velocity there.

\section{Numerical implementation}

A finite-difference discretization of differential problem was performed. Firstorder derivative of $u$ in eqns (11) and (12) were replaced by their central approximations. 
The integration domain was discretized in rectangular-shaped cells, of size $\Delta z \times$ $\Delta y$. Continuous functions $u, \rho$ become discrete functions, pertinent to the centre of gravity of cells. Therefore they have been implemented as matrices. Similarly functions $\tau_{y x}$ and $\tau_{z x}$ are implemented as matrices, with the following convention: $\tau_{z x}(i, j)$ is the shear stress acting over the right-hand side of the cell $(i, j)$, and $\tau_{y x}(i, j)$ is the shear stress acting over the lower side of the cell $(i, j)$. Stresses are regarded as positive when concordant with $x$-axis.

A scheme of conventions about $\tau$ stresses is reported in fig. 3 .

Therefore, instead of a momentum equation, a forces balance can be written for the generic cell $(i, j)$ :

$$
\begin{aligned}
& {[g \rho(i, j) \sin \theta]+\left[\tau_{z x}(i, j)-\tau_{z x}(i, j-1)\right] / \Delta z+} \\
& {\left[\tau_{y x}(i, j)-\tau_{y x}(i-1, j)\right] / \Delta y=0 .}
\end{aligned}
$$

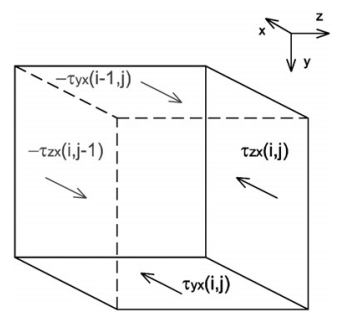

Figure 3: The convention assumed for $\tau$ stresses.

There are as many equations as are the cells, and so, as are unknown variables $u(i, j)$. A $64 \times 64$ cells discretization has been used: the differential problem has been changed in a non-linear system of 4096 equations. Boundary conditions have been implemented, by using ghost null values of $u$ at solid boundary and by imposing $\tau_{y x}=0$ at free surface.

To solve the non-linear system, it was used "Fsolve", which is a trust-region algorithm, included in Optimization Toolbox [6]. Solution tolerance was set to $10^{-6}$. To improve performances, the symmetry of the problem was exploited (for the non-linear system to have only 2048 equations) and it was used a pattern matrix, which informs the computer about zeros, in order to obtain a faster computation of Jacobian matrix.

\subsection{Loops to define $c$ matrix and convergence of algorithm}

Every time the algorithm solves the differential problem, a guessing distribution of $c$ is assumed. The computation finishes when the solution is congruent with its distribution of $c$. It is possible to state two different congruence conditions: 
- "threshold velocity condition", which is verified when the velocity solution is smaller than threshold velocity in cells where $c=c_{\max }$ and only in them;

- "flow concentration condition", which is verified when $c_{f}$, calculated since the differential problem for $u$ has been solved, is equal to $c_{f}$ to simulate.

The second condition is used to assure that the specific motion observed in laboratory flume experiments is simulated and not any other.

Outside of the code which solves the differential problem, there are two nested do-while loops, responsible to verify the congruence conditions: the outer one is pertinent to the "flow concentration condition", the inner one to the "threshold velocity condition". The hypothesis that $c_{m}$ is constant in every columns of the cross section is assumed. Therefore, to fix a local concentration distribution, there are $n+1$ freedom degrees, where $n$ is the number of columns (in the case of this work 64): a degree is $c_{m}$ and the $n$ others ones are the packing positions in each column, i.e. the positions where $c$ becomes equal to its maximum value $c_{\max }$.

The flowchart of algorithm is reported in fig. 4.

Having obtained the $u$ solution, the flow rate $Q$, which is useful for the analysis of results, has been calculated.

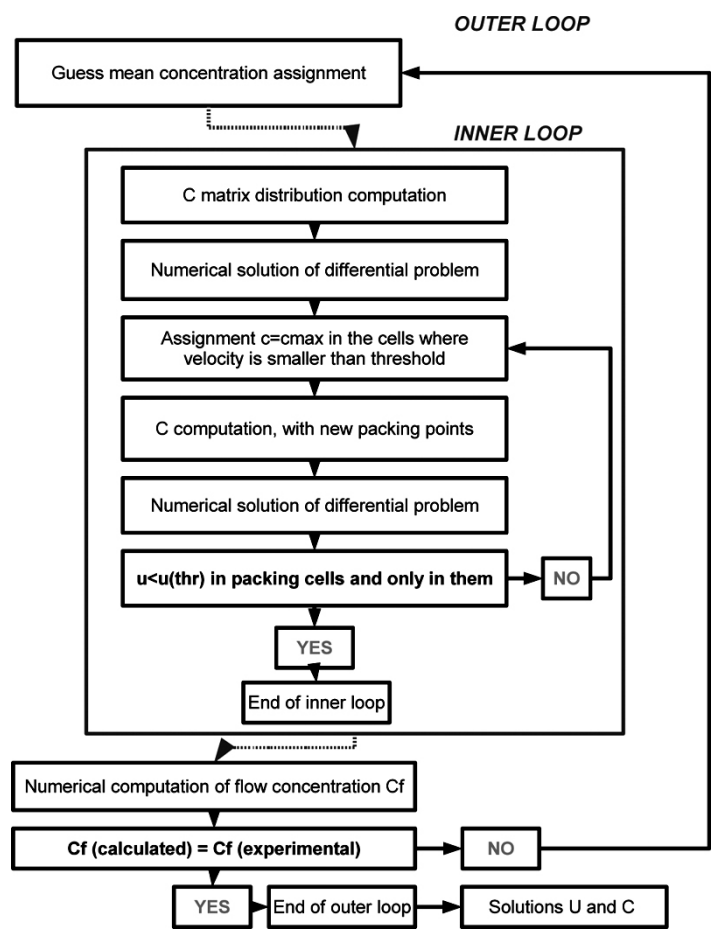

Figure 4: Algorithm flowchart. 
Due to loops outside of differential problem, which can be regarded as Turingcomputable functions but not analytical, it is very difficult to obtain a strict proof of convergence, by using spectral methods. An heuristic way has been tackled: it has been observed that even increasing the discretization level (up to $160 \times 160$ cells) the solution weakly changes. Furthermore, the solution seems to be independent of first guessing concentration distribution [7].

\section{Analysis of results and conclusions}

Velocity and local concentration distributions, obtained by the simulation, are reported in fig. 5. There is a discontinuity of the first type in $c$ distribution: it is, of course, a loophole of the model, on which future studies will be focused.
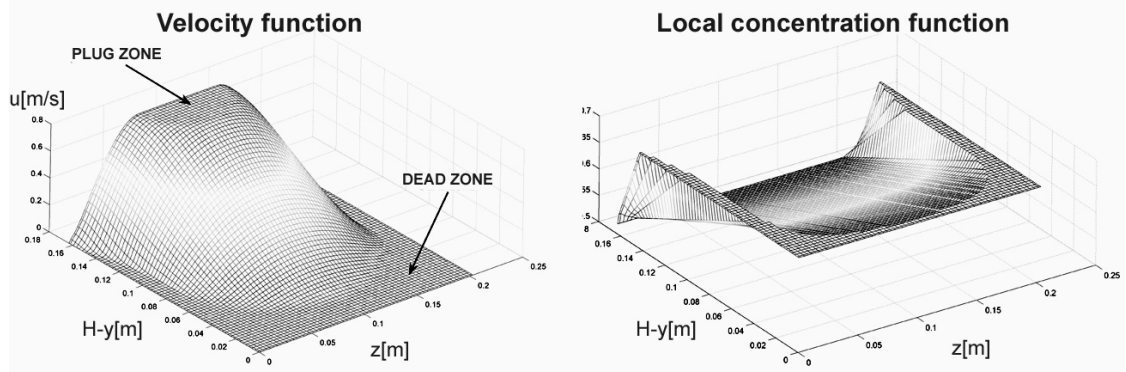

Figure 5: Velocity and local concentration distributions obtained by the simulation: constant velocity zones are highlighted.

Although a modified rheological law with a rather big value of $\epsilon^{2}\left(10^{-3}\right)$ has been used, a velocity distribution with well defined dead and plug zones can be easily seen (fig. 5): in other words, the integration method seems to be suitable for this kind of problems.

The expected boundary points of plug and dead zones, for the generic column $j$, occur when the total shear stress is equal to the yield stress:

$$
\sqrt{\tau_{y x}^{2}+\tau_{z x}^{2}}=\tau_{B}(c)
$$

It is very interesting, now, to compare boundary points of constant velocity zones, which can be observed in velocity distribution after simulation, with the expected boundary points, for each column of integration. The remarkable result of this work is that, everywhere in the integration domain, one can notice an encouraging correspondence between these points. Fig. 6 reports the comparison at middle column of the flume. That is a further confirmation that this modifiedrheology numerical method seem to be working, even with high values of constant $\epsilon^{2}$. 

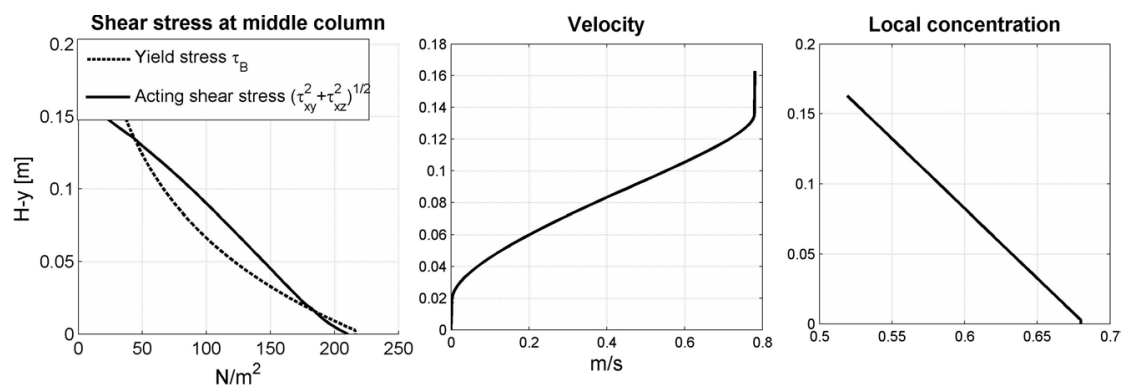

Figure 6: Comparison among total stress, yield stress, velocity distribution and local concentration.

The model needs two parameters, $c_{\max }$ and $u_{t h r}$, not yet obtainable by direct measurements. So they have to be fixed, by a back-analysis of some laboratory flume experiments.

In future research simulated flow rates with experimental measures will be compared and the condition of threshold velocity will be improved, with the contribution of further experimental results. Moreover, it will be interesting to run the model in slip condition at solid boundary and, hopefully, to implement Coulombian stresses, which seem to be not totally negligible in the dead zones.

\section{References}

[1] Macosko, C.W., Rheology - Principles, Measurements and Applications. Wiley-VCH: New York, 1994.

[2] Dalrì, C., Fraccarollo, L., Larcher, M. \& Armanini, A., Analisi sperimentale per la caratterizzazione del flusso di miscugli iperconcentrati di origine sintetica e naturale. Proceedings of XXIX Convegno Nazionale di Idraulica e Costruzioni Idrauliche - IDRA, Trento, 2004.

[3] Whipple, K.X., Open channel flow of bingham fluids: application in debrisflow research. Journal of Geology, 105, pp. 243-263, 1997.

[4] Bercovier, M. \& Engleman, M., A finite element method for incompressible non-newtonian flows. Journal of Computational Physics, 36, pp. 313-326, 1980.

[5] Martino, R. \& Papa, M.N., Effetto delle pareti nelle correnti detritiche: primi risultati. Proceedings of XXIX Convegno Nazionale di Idraulica e Costruzioni Idrauliche - IDRA, Trento, 2004.

[6] AA.VV., Optimization toolbox - Trust-region methods for nonlinear minimization. Matlab 70 R14 Help, 2004.

[7] Sarno, L., Simulazione di una colata di fango in regime visco-plastico e condizioni di moto uniforme. Degree thesis - Naples University, Naples, 2006. Supervisor: Martino R. 